\title{
Introducción de Matlab en la docencia de ingeniería nuclear. Caso práctico: estudio de la cinética puntual
}

\section{N. Olmo-Juan ${ }^{\mathrm{a}}$, T. Barrachina ${ }^{\mathrm{b}}$, R. Miróc , J. Sancho $^{\mathrm{d}}$ y G. Verdú}

${ }^{a}$ Dpto. de Ingeniería Química y Nuclear. Universitat Politècnica de València. nioljua@iqn.upv.es, bDpto. de Ingeniería Química y Nuclear. Universitat Politècnica de València. tbarrachina@iqn.upv.es, 'Dpto. de Ingeniería Química y Nuclear. Universitat Politècnica de València. rmiro@iqn.upv.es, ${ }^{d}$ Dpto. de Ingeniería Química y Nuclear. Universitat Politècnica de València. jsancho@iqn.upv.es, eDpto. de Ingeniería Química y Nuclear. Universitat Politècnica de València. gverdu @iqn.upv.es.

\section{Abstract}

One of the great challenges to be tackled in the teaching and teaching of subjects related to the intensifications of the degrees and masters is the development of tools and procedures that allow the student to achieve a deep learning of the complex concepts studied in classroom theory classes. .

This effort is in most cases contemplated within the time allocated in the development of each subject to the practices, whether they are performed in a laboratory, a computer laboratory or, even, in classroom practices. These practices, properly focused, are a powerful tool, both for the teacher and the student, because they allow to overcome the limitations existing in the theoretical classes that continue to be mainly master classes.

In the present work, the teaching innovation introduced in the teaching of a practice is detailed, for which a methodology has been defined and which has been implemented using the Matlab computer tool, which allows to deepen the foundations of the equations for the calculation of the neutron population in a nuclear reactor and its variation over time in the simplification of point kinetics. These contents are common to different subjects of degree and master so this innovation has an extensive application.

Keywords: point kinetic, Matlab, teaching innovation

\section{Resumen}

Uno de los grandes retos a abordar en la docencia e impartición de asignaturas correspondientes a las intensificaciones de los grados y másteres es el desarrollo de herramientas y procedimientos que permitan al alumno alcanzar un aprendizaje profundo de los conceptos complejos estudiados en las clases de teoría de aula.

Dicho esfuerzo es en la mayoría de los casos contemplado dentro del tiempo destinado en el desarrollo de cada asignatura a las prácticas, ya sean desempeñadas en un laboratorio, una sala informática o, incluso, en las prácticas de aula. Estas prácticas, enfocadas adecuadamente, son una poderosa herramienta, tanto para el docente como el alumno, pues permiten superar las limitaciones existentes en las clases teóricas que siguen siendo mayoritariamente clases magistrales. 
En el presente trabajo se detalla la innovación docente introducida en la impartición de una práctica, para la que se ha definido una metodología y que ha sido implementada haciendo uso de la herramienta informática Matlab, que permite profundizar en los fundamentos de las ecuaciones para el cálculo de la población de neutrones en un reactor nuclear y su variación en el tiempo en la simplificación de la cinética puntual. Estos contenidos son comunes a diversas asignaturas de grado y máster por lo que esta innovación tiene una extensa aplicación.

Palabras clave: cinética puntual, MATLAB, innovación docente

\section{Introducción}

La impartición de docencia en las asignaturas consideradas de intensificación o especializantes, que se ofertan en las fases finales de los grados y másteres, presentan con frecuencia el reto añadido de iniciar al alumnado en un nuevo campo o disciplina, que habitualmente tan sólo le ha sido escuetamente descrito o introducido.

A este contexto también se le puede añadir la necesidad de transmitir al alumno la gran cantidad de conceptos que conforman el proceso de especialización, en un período de tiempo reducido. Esto se traduce en que se intente impartir al alumno una gran cantidad de conocimientos que, en ocasiones, resultan ser densos conceptos que requieren de un sosegado razonamiento y una detenido análisis para los que no se dispone del tiempo material necesario.

La responsabilidad de solventar estas limitaciones corresponde al docente que, haciendo uso de los distintos recursos y facilidades a su alcance, debe superar cualquier restricción que impida que el alumno adquiera los conocimientos que demanda para su futuro desempeño laboral.

Una de las intensificaciones que, por su evidente complejidad, se ajusta enormemente a la situación descrita es la intensificación de energía nuclear. En la misma, se procede a la inmersión del alumno en los conceptos y fenomenología relativos a la física del reactor (Murray, 2017), la termohidráulica, la seguridad nuclear, la protección radiológica e, incluso, la medicina nuclear. Nos encontramos, por tanto, ante una intensificación con un enorme contenido a impartir dentro de la limitación temporal que define su confinamiento al período reservado a la especialización.

Por este motivo, en el presente trabajo, con la finalidad de facilitar el proceso de aprendizaje de los fundamentos de la cinética puntual (Snoj, 2011) de los reactores nucleares, se describe la metodología seguida en la impartición de una práctica informática. En la misma se han incluido diversas mejoras que tienen como finalidad mejorar el soporte disponible para el docente e incrementar el protagonismo del alumno durante la actividad.

Para ello, se ha utilizado una herramienta informática que los alumnos ya han trabajado en cursos anteriores como es Matlab (Giménez et al., 2016). Además es un recurso que los alumnos tienen disponible a través de licencia universitaria.

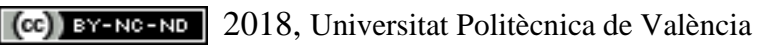

Congreso IN-RED (2018) 
Este artículo se organiza de la siguiente manera: en primer lugar se detallan los objetivos principales así como, el contexto y fundamentos teóricos que justifican el desarrollo de esta metodología. A continuación, se presenta con detalle el desarrollo de la innovación implementada. Finalmente, se presentan los resultados obtenidos y las principales conclusiones.

\section{Objetivos}

Los objetivos de este trabajo son dos:

- Testar el comportamiento descrito por las ecuaciones que rigen la cinética puntual.

- Valorar la influencia que poseen diversas fuentes de realimentación en la inserción de reactividad en la fenomenología de un reactor

\section{Justificación de la innovación}

Se detalla a continuación el contexto en el que se enmarca la innovación docente considerada y una breve descripción de las particularidades que presentan las ecuaciones de la cinética puntual.

\subsection{Contexto}

La metodología introducida pretende servir como ayuda al profesor que desea que el alumno profundice en su comprensión de la cinética puntual. Por este motivo, el aprendizaje del alumno como fin último del proceso, se ha concebido a éste como protagonista destacado de todo el proceso.

La innovación docente implementada ha sido diseñada con la intención de ser completamente válida para la impartición de una práctica informática, independientemente de que la asignatura en que sea programada esté contenida de un grado o máster.

Esta versatilidad nos resulta de gran interés puesto que nos permite disponer de una práctica "modular" perfectamente definida y debidamente validada para su incorporación en el programa de cualquier asignatura en que la cinética puntual sea tratada.

De esta forma, se prevé que pueda resultar de gran utilidad su incorporación a las asignaturas:

- Operación de reactores nucleares. $4^{\circ}$ curso del Grado en Ingeniería de la Energía.

- Tecnología Nuclear. $3^{\circ}$ curso del Grado en Ingeniería de la Energía.

- Dinámica Nuclear. $1^{\circ}$ curso del Máster Universitario en Seguridad Industrial y Medio Ambiente.

- Energía Nuclear y radiaciones. $2^{\circ}$ curso del Máster Universitario de Ingeniería Industrial.

Con la finalidad de verificar la idoneidad de las distintas actividades contempladas y la adopción, en caso de ser necesarias, de medidas correctoras; se ha incluido, inicialmente, la

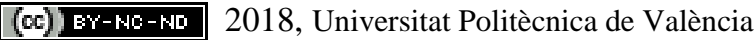


práctica en la programación de la asignatura de Operación de reactores nucleares del presente curso. Dado el buen desempeño obtenido se espera su implementación en el resto de las asignaturas en la próxima edición.

\subsection{La cinética puntual}

Las ecuaciones de la cinética puntual son una aproximación clásica de la ecuación de transporte neutrónico dependiente del tiempo y las ecuaciones de la densidad de precursores (Bell and Glasstone, 1970). Las hipótesis que se asumen y bajo las que podemos emplear dicha aproximación son la inexistencia o escasa relevancia de la influencia espacial dentro del reactor en los transitorios contemplados, así como en una reducida duración estimada de los mismos.

De esta forma, se llega a un sistema de ecuaciones simplificado en el que sólo se tiene en cuenta la evolución de la reactividad debida a la densidad neutrónica, pudiéndose considerar uno o dos grupos de energía, y el acoplamiento de la misma con la densidad de precursores de neutrones diferidos. La elección de sólo un grupo o de diversos grupos de precursores, con la influencia que dicho nivel de detalle posee en la inercia del sistema frente a los cambios, conduciría a la definición de un sistema que comprendería entre 2 ecuaciones acopladas, en su caso más simplificado, y 8 ecuaciones, en aquellos casos en que se necesite un mayor nivel de detalle.

Sin embargo, pese a la relativa complejidad que puede llegar a alcanzar el sistema de ecuaciones, las ecuaciones de la cinética puntual son mucho más simples que las correspondientes a la ecuación de difusión o del transporte, lo que permite que su resolución sea claramente más sencilla dado que existe una solución directa del sistema de ecuaciones. Por este motivo, su resolución se ve simplificada y permite su implementación en un entorno de desarrollo, como Matlab.

La implementación del sistema de ecuaciones en una herramienta de software matemático, no sólo aporta la obtención de la solución del modelo planteado sino que también proporciona un método de resolución rápido, lo que permite al alumno modificar los valores de los distintos parámetros de entrada. Con ello, se facilita la comprensión del alumno de la influencia e importancia de cada una de las variables.

Por último, se ha introducido en el programa un modelo termohidráulico simplificado que nos permitirá, si así lo deseamos, tener en cuenta la influencia de la realimentación termohidráulica ante un suceso de inserción de reactividad.

\section{Desarrollo de la innovación}

Con la finalidad de despertar el interés del alumno se ha contemplado el desarrollo como un acto dividido en tres partes. Se pretende con ello lograr una actitud por parte del alumnado lo más activa posible, evitando que el desempeño de la actividad quede confinada en exclusividad a la duración de la práctica.

(c) EY-NG-ND 2018, Universitat Politècnica de València

Congreso IN-RED (2018) 
Pese a que la innovación docente presentada comprende tres etapas diferenciadas, como son las tareas previas a la práctica, el desempeño de la misma y la actividad posterior a ella; cabe recordar que existe una etapa previa indispensable a todo el proceso que es, la enseñanza de los principios teóricos en el aula. Dicha introducción, en su desempeño óptimo, debe ser impartida preparando, además, al alumno para lo que verá en la práctica. Lográndose así la sinergia en la que las actividades teóricas y prácticas se tratan perfectamente alineadas.

Sin embargo, no es el objetivo de esta innovación docente determinar cómo debe ser la cinética puntual tratada en las prácticas de aula, por lo que respecto a esta fase previa tan sólo se comentarán las puntualizaciones ya realizadas.

Procedemos seguidamente a la descripción cronológica de las actividades contempladas en la innovación:

\subsection{Actividad previa a la práctica}

Antes de realizar la práctica se le envía al alumno un guión de la práctica, junto con el recordatorio de la misma. Aunque las fechas, horarios y aulas están disponibles desde el inicio del curso en el sitio web de la Escuela, se considera muy adecuado enviar un recordatorio unos días antes, a modo de recordatorio, pero también, como una forma de contextualizar al alumno en la práctica que en pocos días realizará.

Dicho documento previo tiene la finalidad, por tanto, de indicar al alumno que contenidos teóricos serán enlazados en la práctica y como se desempeñará la misma. Así, en un documento breve, de 6 páginas, para no saturar al alumno y que éste prescinda de su lectura; se realiza una breve introducción a los contenidos teóricos, se detallan todas las variables que se emplearán junto con su significado y se explica cómo están programadas las funciones. Al final del documento también se presentan las actividades que serán realizadas y su forma de resolución. El hecho de que el alumno pueda ver como ejecutar las tareas planteadas y su resolución, no nos supone ningún inconveniente. Al contrario, lo que queremos es que el alumno realice un pensamiento crítico del resultado y valore el peso de cada factor en la respuesta final.

\subsection{Desempeño de la práctica}

Por lo que respecta a las tareas realizadas durante la práctica, se presenta la descripción de la impartición de la misma, de su implementación en Matlab. Aunque la duración de la práctica podría adaptarse a los requisitos del profesor, número de alumnos, etc. la duración inicial considerada es de 2 horas.

\subsubsection{Impartición de la práctica}

$\mathrm{Al}$ inicio de la práctica se comprueba que los alumnos hayan leído el previo y entendido correctamente, solventándose las dudas que pudieran ser expuestas. A continuación, se 
proyecta una presentación que vuelve a enlazar con las ecuaciones expuestas en clase, así como la fenomenología asociada y que será vista en la práctica.

Cabe destacar que este inciso teórico, que viene a refrescar lo ya visto en clase y en la actividad previa, difiere en su alcance y presentación del documento previo ya comentado. Dicha divergencia tiene como finalidad que el alumno que ha realizado el previo, tal y como se le ha pedido, no considere que se le está repitiendo de nuevo el mismo contenido. Pero, por otra parte, se considera indispensable dicho inciso para asegurarnos de que todos los alumnos, incluso los que no hayan realizado la lectura previa, partan desde el punto deseado.

A lo largo de la presentación, se realizarán preguntas a los alumnos, buscando activar su participación activa en la práctica y evaluando, de paso, si están procesando y razonando los contenidos tal y como el docente desea.

Una vez finalizada la presentación, se procede a la descripción de la implementación de las ecuaciones realizada en Matlab, su explicación y su funcionamiento. En el siguiente apartado serán descritas en de talle.

Una vez que los alumnos han entendido el funcionamiento de la práctica, se les plantean 3 ejercicios, ya presentados en el previo, que deben realizar. La ejecución de los mismos es realizada por toda la clase a la vez ya que se concibe la ejecución en Matlab como un medio con el que visualizar el comportamiento de la cinética puntual, siendo la finalidad la correcta comprensión de éste y la influencia que en él tienen distintos fenómenos también vistos.

Los ejercicios consisten en modificaciones de los parámetros de entrada y el análisis de su influencia en la salida, así como en el uso de modelos que resuelven el sistema de ecuaciones considerando el reactor como un ciclo abierto o cerrado, teniendo en cuenta en éste caso la influencia de la fenomenología asociada a un suceso de inserción de reactividad.

La clase finalizará cuando se hayan realizado todas las actividades y el docente considere que los alumnos han superado debidamente los objetivos contemplados.

\subsubsection{Implementación en Matlab}

La implementación del sistema de ecuaciones en Matlab se ha realizado mediante la creación de diversas funciones que serán invocadas según el caso de problema que se desee resolver. No se ha considerado el uso de la herramienta Matlab GUIDE ya que se ha intentando realizar un programa lo más modular y comprensible posible, para el alumno como para docentes no familiarizados con el uso de dicha herramienta.

De esta forma, se han escrito 8 funciones diferentes en Matlab que permiten al alumno trabajar directamente con la fuente y además sectorizar debidamente la la tarea realizada en cada una de ellas.

La mitad de estas funciones tienen como finalidad devolver el valor del parámetro de interés (Reactividad, existencia de una fuente de neutrones, temperatura del combustible o temperatura del refrigerante) para cada paso de tiempo. Es decir, nos permitirían obtener el resultado para un caso en el que algunos de estos parámetros fluctuara en el tiempo.

(cc) EY-NC-ND 2018, Universitat Politècnica de València

Congreso IN-RED (2018) 
Por otra parte, el resto de funciones permiten la resolución del sistema de ecuaciones según se desee resolverlo considerando el modelo en bucle abierto o cerrado, con realimentación termohidráulica. Para cada caso, se contemplan dos funciones conteniendo una, la principal, el procesamiento de las variables de entrada, la llamada a las 4 funciones de parámetros relevantes, así como la metodología idónea para la resolución del sistema de ecuaciones correspondiente y la impresión de los resultados; mientras que la otra contiene el sistema de ecuaciones que será invocado para su resolución.

Son como puede imaginarse, una serie de funciones relativamente sencillas y simplificadas que permiten tratar de forma independiente cada parámetro y sistema de ecuaciones. El método de resolución empleado para cada uno de los modelos, abierto o cerrado, es el método Runge-Kutta de segundo orden (ode45) y el método de orden variable de Adams-BashforthMoulton (ode113), respectivamente.

La forma de ejecutar cada uno de los modelos parte de la definición en el escritorio principal de Matlab de las variables que serán transmitidas en la llamada a la función deseada. Una vez especificadas e invocada la función principal, correspondiente al modelo en bucle abierto o cerrado, el usuario obtendrá directamente las gráficas de la evolución de los parámetros de interés para cada modelo, concentración de neutrones y de precursores de neutrones diferidos para el sistema abierto; a los que se les añade la evolución de las temperaturas del combustible y el refrigerante para el sistema cerrado.

En caso de desear modificar alguno de los parámetros de entrada bastará con modificarlos en la llamada inicial a la función correspondiente si no son uno de los 4 parámetros de mayor relevancia, o bien en el interior de sus respectivas funciones.

Por lo que respecta a la elección de un modelo de sistema abierto o cerrado basta con invocar a la función principal correspondiente, habiendo definido adecuadamente las variables de entrada necesarias para cada caso.

\subsection{Actividad posterior}

Una vez finalizada la práctica no se considera indispensable la realización de un trabajos posterior. En la mayoría de las asignaturas el número de alumnos es reducido y el seguimiento de éstos es cercano.

Sin embargo, si que puede resultar interesante solicitar, en aquellos grupos en el que el número de alumnos sea considerable, la realización de una memoria en la que el alumno detalle como hacer uso de la implementación en Matlab y un análisis de los resultados vistos y otros sugeridos, incluyendo un análisis de sensibilidad de determinados parámetros que son de interés en el control del reactor.

De esta forma, nos aseguraríamos que el alumno ha entendido correctamente el funcionamiento y ejecución de la implementación en Matlab, así como que es capaz de evaluar la influencia e importancia que cada parámetro posee.

(cc) EY-NC-ND 2018, Universitat Politècnica de València 


\section{Resultados}

Como se ha comentado en el apartado en que se ha contextualizado la innovación docente, la impartición descrita ha sido abordada inicialmente en la programación de una única asignatura, Operación de reactores nucleares. La elección de esta asignatura se ha basado en el gran peso que en su contenido posee la cinética puntual, así como el número reducido de alumnos que nos permiten tener una realimentación crítica constante debida al trato cercano entre el alumno y el profesor.

Así, los resultados obtenidos acerca de la introducción de la innovación docente y su eficacia, han sido obtenidos directamente consultando a los alumnos y observando su evolución.

Uno de los aspectos que mejor han sido valorados ha sido la óptima ubicación de la práctica en la programación de la asignatura. La práctica ha sido realizada tras iniciar el estudio de la cinética puntual y antes de finalizar dicho bloque. Lo que ha permitido al alumno la introducción preliminar de los conceptos, su visualización durante la práctica $\mathrm{y}$, posteriormente, la recapitulación de éstos en clase.

Otros factores que han recibido buena acogida ha sido la elección de la herramienta Matlab como herramienta informática. Los alumnos ya conocen este programa de anteriores asignaturas que, además, puede ser descargado desde el repositorio de software que la Universidad pone a disposición de los alumnos.

Sin embargo, se ha constatado que no todos los alumnos realizaron la lectura previa a la práctica, por lo que deberá realizarse alguna corrección en la metodología para lograr una mayor implicación del alumno en todas las fases consideradas.

\section{Conclusiones}

Se ha llevado a cabo la implementación de una innovación docente para mejorar la impartición de la cinética puntual. Para ello se ha creado una metodología completa que define desde las actividades previas a la práctica, como su desempeño y su posible memoria posterior.

Además, la innovación ha sido basada en el uso de la herramienta de software matemático, Matlab. Esta elección nos permite no sólo la resolución del sistema de ecuaciones planteado sino también una vérsatil en la que poder realizar, con facilidad, modificaciones de los valores correspondientes a los distintos parámetros de entrada. Además, es una herramienta con la que el alumno se encuentra plenamente familiarizado.

Los resultados obtenidos han sido satisfactorios, considerándose su implementación de gran ayuda para el docente y suponiendo su correcta programación y ejecución, de gran ayuda para el aprendizaje del alumnado.

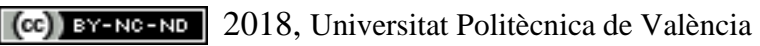

Congreso IN-RED (2018) 


\section{Trabajos futuros}

Pese al buen desempeño y satisfactorio resultado de la innovación docente, en el marco de un proceso de mejora contínua, diversos aspectos pueden ser señalados.

Con el objetivo de mejorar la participación del alumno en el desempeño de la práctica, se considera interesante lograr aumentar la motivación del alumno, que le lleve a disponer una actitud más proactiva hacia el aprendizaje a lo largo de todas las fases consideradas. Un buen ejemplo de ello sería que todos los alumnos realizasen la actividad previa contemplada.

Por lo que respecta a la implementación de la práctica en Matlab, se contempla la posibilidad de mejorar el proceso de ejecución de la misma, así como la presentación de los resultados. Se señala como una posible mejora la reescritura del programa haciendo uso de la herramienta Matlab GUIDE.

\section{Referencias}

BELL, G.I.., y GLASSTONE, S. (1970). Nuclear reactor theory. New York, U.S.: Van Nostrand Reinhold Inc.,U.S.

GIMÉNEZ, F., MONSORIU, F.A. y ABRAHAM, S. (2016). " Aprender métodos matemáticos programando con Matlab" en Congreso In-Red 2016. Valencia, pp. 276-284. Disponible en: http://ocs.editorial.upv.es/index.php/INRED/INRED2016/paper/viewFile/4430/2000 [Consulta: $25 \mathrm{de}$ marzo de 2018]

MURRAY, R.L., (2017). "Reactor Kinetics Pedagogical Insight" en Nuclear science and Engineering. $118: 4,268-271$.

SNOJ, L. et al, (2011). "Advanced methods in teaching reactor physics" en Nuclear science and Engineering. 241 (4), 1008-1012.

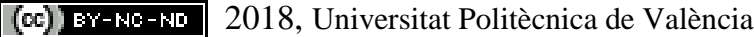

